\title{
Structured sparse $K$-means clustering via Laplacian smoothing
}

\author{
Weikang Gong a,b,*, Renbo Zhao ${ }^{c}$, Stefan Grünewald ${ }^{\mathrm{a}}$ \\ ${ }^{a}$ Key Laboratory of Computational Biology, CAS-MPG Partner Institute for Computational Biology, Shanghai Institutes for Biological Sciences, Chinese \\ Academy of Sciences, Shanghai, China \\ ${ }^{\mathrm{b}}$ University of Chinese Academy of Sciences, Beijing, China \\ ${ }^{\mathrm{c}}$ Department of Industrial Systems Engineering and Management, National University of Singapore, Singapore
}

\section{A R T I C L E I N F O}

\section{Article history:}

Received 25 August 2017

Available online 5 June 2018

\section{Keywords:}

Structured sparse clustering

$K$-means clustering

Feature selection

Graph Laplacian smoothing

\begin{abstract}
A B S T R A C T
We propose a structured sparse $K$-means clustering algorithm that learns the cluster assignments and feature weights simultaneously. Compared to previous approaches, including $K$-means in MacQueen [28] and sparse $K$-means in Witten and Tibshirani [46], our method exploits the correlation information among features via the Laplacian smoothing technique, so as to achieve superior clustering accuracy. At the same time, the relevant features learned by our method are more structured, hence have better interpretability. The practical benefits of our method are demonstrated through extensive experiments on gene expression data and face images.
\end{abstract}

\section{Introduction}

$K$-means algorithm [28] has been one of the most popular clustering algorithms due to its simplicity and intuitive interpretations. Given a set of data samples $\left\{\mathbf{x}_{i}\right\}_{i=1}^{n} \subseteq \mathbb{R}^{d}$, it aims to find a partition of $[n] \triangleq\{1, \ldots, n\}$, denoted as $\left\{\mathcal{C}_{k}\right\}_{k=1}^{K}$, to locally minimize ${ }^{1}$

$\min _{\left\{\mathcal{C}_{k}\right\}_{k=1}^{K}} \sum_{k=1}^{K} \frac{1}{\left|\mathcal{C}_{k}\right|} \sum_{i, i^{\prime} \in \mathcal{C}_{k}}\left\|\mathbf{x}_{i}-\mathbf{x}_{i^{\prime}}\right\|_{2}^{2}$.

Each entry of any data sample $\mathbf{x}_{i}$ is termed as a feature. From (1), one observes that $K$-means makes use of all the features for clustering. However, for high-dimensional data, typically only a (small) subset of features are responsible for clustering different groups. Based on this characteristic, many sparse clustering algorithms $[4,19,31,39,44,46]$ have been proposed to simultaneously cluster (high-dimensional) data and select the relevant features. For a detailed overview of these methods, see e.g., Witten and Tibshirani [46]. Among these algorithms, a promising approach, namely sparse $K$-means clustering, was proposed in Witten and Tibshirani [46]. Specifically, it assigns (nonnegative) weights $\left\{w_{j}\right\}_{j=1}^{d}$ to each feature and enforces sparsity on the weight vector $\mathbf{w} \triangleq\left(w_{1}, \ldots, w_{d}\right)^{T}$ via the $\ell_{1}$-constraint. Formally, it finds $\left\{\mathcal{C}_{k}\right\}_{k=1}^{K}$ and $\mathbf{w}$ by solving the following (nonconvex) optimization

\footnotetext{
* Corresponding author.

E-mail address: weikanggong@gmail.com (W. Gong).

1 Since (1) is NP-hard [1], $K$-means-based algorithms (including the standard one and its variants) can only find a local optimum of (1).
}

problem:

$$
\begin{gathered}
\max _{\left\{\mathcal{C}_{k}\right\}_{k=1}^{K}, \mathbf{w}} \sum_{j=1}^{d} w_{j}\left(\frac{1}{n} \sum_{i, i^{\prime} \in[n]} \Delta_{i, i^{\prime}, j}-\sum_{k=1}^{K} \frac{1}{\left|\mathcal{C}_{k}\right|} \sum_{i, i^{\prime} \in \mathcal{C}_{k}} \Delta_{i, i^{\prime}, j}\right) \\
\text { s. t. }\|\mathbf{w}\|_{1} \leq \bar{s},\|\mathbf{w}\|_{2}^{2} \leq 1, w_{j} \geq 0, \forall j \in[d] .
\end{gathered}
$$

In (2), $\Delta_{i, i^{\prime}, j} \triangleq\left(x_{i, j}-x_{i^{\prime}, j}\right)^{2}$ and the tuning parameter $\bar{s}>0$ controls the sparsity of $\mathbf{w}$. With a suitable $\bar{s}$, this method leads to a significant improvement in the clustering accuracy compared to the original $K$-means algorithm $[19,46]$.

However, in the sparse $K$-means algorithm, the correlations among features are not exploited. Indeed, one observes that for fixed clusters $\left\{\mathcal{C}_{k}\right\}_{k=1}^{K}$, the problem (2) is separable across the coordinates of $\mathbf{w}$ (in both objective function and constraints). In many scenarios, the relevant features learned by such an approach may not be structured, hence degrading their interpretability and in turn, clustering accuracy. To be specific, let us consider a toy example. We first generated four images, each containing an identical solid square at different locations with pixel intensity one. For each image, we replicated it to obtain 25 copies and added white noise to each copy. We further smoothed every copy via a Gaussian kernel. As a result, we obtained four classes of images, whose averages are shown in Fig. 1(a)-(d). We then applied sparse $K$-means and our method (which can incorporate information about feature correlations) to cluster these images. The pixel weights learned by sparse $K$-means and our method are shown in Fig. 1(e) and (f) respectively. We observe that the relevant pixels identified by sparse $K$-means are scattered and overly parsimonious. Indeed, we can hardly observe any meaningful patterns from them. 


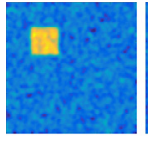

(a)

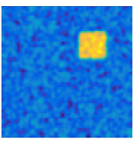

(b)

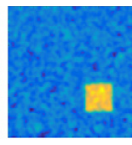

(c)

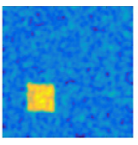

(d)

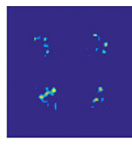

(e)

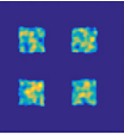

(f)
Fig. 1. A toy example. Images (a) to (d) show the averages of four classes of noisy images respectively. Images (e) and (f) show the pixel weights learned by sparse $K$-means and our method respectively.

In stark contrast, our method effectively identifies all of the four solid squares, which clearly differentiate the four classes of images. Consequently, our method achieves a much higher clustering accuracy-the Rand Indices [32] of the clustering results by sparse $K$-means and ours are 0.87 and 1.00 respectively. In Section 4, we will show the improvements made by our method are also significant on a wide range of real data.

\subsection{Main contributions}

We propose a structured sparse K-means clustering algorithm by incorporating the correlation information among features via the Laplacian smoothing technique [13]. To the best of our knowledge, this is the first algorithm that exploits feature correlations in sparse clustering. Previously, the Laplacian smoothing technique has been used in several applications, including classification from labeled and unlabeled data [7-9,48], whole-brain prediction analysis [21] and online dictionary learning [16]. We apply this technique to sparse $K$-means clustering in an innovative manner, and develop an efficient algorithm to learn the cluster assignments and weight vector simultaneously. We show, via extensive experiments on gene expression data and face images, that compared to sparse $K$-means, our approach can significantly improve both the clustering accuracy and the interpretability and meaningfulness of the learned features. In addition, our approach can be easily extended to the case where the loss function is beyond the squaredEuclidean loss. See Section 3.2 for details.

\subsection{Notations}

We use lowercase, bold lowercase and bold uppercase letters to denote scalars, vectors and matrices respectively. For a vector $\mathbf{x}, \mathbf{x} \geq 0$ denotes entrywise nonnegativity. We use $x_{i}$ and $x_{i j}$ to denote the $i$ th and $(i, j)$ th entry of a vector $\mathbf{x}$ and a matrix $\mathbf{X}$ respectively. We define $\mathbb{R}_{+} \triangleq[0,+\infty), \mathbb{N} \triangleq\{1,2, \ldots\}$ and $C(n, k) \triangleq$ $n ! /((n-k) ! k !)$, for any $n, k \in \mathbb{N}, n \geq k$.

\section{Problem formulation}

To begin with, consider an undirected weighted graph $\mathcal{G} \triangleq$ $(\mathcal{V}, \mathcal{E}, \mathbf{A})$, where the set of nodes $\mathcal{V} \triangleq[d]$, the set of edges $\mathcal{E} \subseteq$ $\{(i, j): i, j \in[d], i<j\}$ and the (symmetric) adjacency matrix $\mathbf{A} \in$ $\mathbb{R}_{+}^{d \times d}$ with $a_{i j}>0$ if $(i, j) \in \mathcal{E}$ and 0 otherwise. In our context, we can use $\mathcal{G}$ to model the relations among the $d$ features. Specifically, each node corresponds to one feature and the weight $a_{i j}$ indicates the correlation between features $i$ and $j$. Based on $\mathbf{A}$, we can define the Laplacian (matrix) of $\mathcal{G}$, denoted as $\mathbf{L}$, such that for any $i, j \in[d]$, $l_{i i} \triangleq \sum_{i^{\prime} \neq i} a_{i i^{\prime}}$ and $l_{i j} \triangleq-a_{i j}$ if $i \neq j$. To learn the cluster membership $\left\{\mathcal{C}_{k}\right\}_{k=1}^{K}$ and weight vector $\mathbf{w}$, we propose to solve the following optimization problem:

$$
\begin{aligned}
& \min _{\left\{\mathcal{C}_{k}\right\}_{k=1}^{K}, \mathbf{w}}-\sum_{j=1}^{d} w_{j}\left(\frac{1}{n} \sum_{i, i^{\prime} \in[n]} \Delta_{i, i^{\prime}, j}-\sum_{k=1}^{K} \frac{1}{\left|\mathcal{C}_{k}\right|} \sum_{i, i^{\prime} \in \mathcal{C}_{k}} \Delta_{i, i^{\prime}, j}\right)+\lambda \mathbf{w}^{T} \mathbf{L w} \\
& \text { s.t. } \mathbf{w} \in \mathcal{W} \triangleq\left\{\mathbf{w} \in \mathbb{R}_{+}^{d}:\|\mathbf{w}\|_{1} \leq s\right\},
\end{aligned}
$$

where $\lambda>0$ is the regularization weight. In (3), we replace the constraint $\|\mathbf{w}\|_{2}^{2} \leq 1$ in (2) with the regularizer $\lambda \mathbf{w}^{T} \mathbf{L w}$. To see the effect of this regularizer, we rewrite it as

$\lambda \mathbf{w}^{T} \mathbf{L w}=\lambda \sum_{i, j: a_{i j} \neq 0} a_{i j}\left(w_{i}-w_{j}\right)^{2}$.

Thus for a fixed $\lambda$, a strong correlation between features $i$ and $j$ (i.e., a large value of $a_{i j}$ ) will lead to a small difference between weights $w_{i}$ and $w_{j}$. For this reason, the technique that uses $\lambda \mathbf{w}^{T} \mathbf{L w}$ as a regularization term is termed Laplacian smoothing [7,13,34]. As a result, if a set of correlated features are relevant for clustering, they tend to be selected together. Similarly, if they are not relevant for clustering, they tend to be discarded together. As shown in our experiments (see Section 4), this leads to greatly improved clustering performance in many cases.

Remark 1. Another popular way to enforce both sparsity and smoothness on the weight vector $\mathbf{w}$ is through the graph fused lasso regularization $[40,47]$. Namely, in (3), $\lambda \mathbf{w}^{T} \mathbf{L w}$ is replaced with $\lambda \sum_{\left(i, i^{\prime}\right) \in \mathcal{E}}\left|w_{i}-w_{i^{\prime}}\right|$. However, this approach has two drawbacks. First, this regularizer implies that each edge in the graph $\mathcal{G}$ has the same weight, which is less flexible compared to our approach. The second drawback is due to the optimization difficulties. Specifically, the proximal operator of the fused lasso regularizer is much more expensive to compute compared to the gradient of $\lambda \mathbf{w}^{T} \mathbf{L w}$ (which is simply $2 \lambda \mathbf{L w}$ ).

\section{Algorithm}

Due to the NP-hardness of (3), finding its global optimum is out-of-reach. To approximately solve (3), we adopt an alternating minimization approach. The details are shown in Algorithm 1. Note that in step (i), the weight $w_{j}^{t}$ in (6) can be absorbed into $\Delta_{i, i^{\prime}, j}$, so that standard $K$-means can be applied. In step (ii), due to the positive semidefiniteness of the Laplacian $\mathbf{L}$ [27], the problem in (7) is convex. We can solve this problem by simply using the gradient projection method [11, Section 2.3], with a constant step size $\left(\lambda\|\mathbf{L}\|_{2}\right)^{-1}$. Specifically, let $\left\{\tilde{\mathbf{w}}_{t}^{i}\right\}_{i \in \mathbb{N}}$ denote the sequence of iterates generated by this algorithm for solving (7). Then the iteration $i$ of this algorithm takes the form

$\mathbf{w}_{t}^{i+1}:=\Pi_{\mathcal{W}}\left[\mathbf{w}_{t}^{i}-\left(\lambda\|\mathbf{L}\|_{2}\right)^{-1}\left(2 \lambda \mathbf{L} \mathbf{w}_{t}^{i}-\boldsymbol{\alpha}^{t+1}\right)\right]$,

where $\Pi_{\mathcal{W}}$ denotes the (Euclidean) projection operator onto $\mathcal{W}$. Since the set $\mathcal{W}$ is the nonnegative $\ell_{1}$-ball, projection onto it can be decomposed into two successive steps, i.e., projection onto the nonnegative orthant $\mathbb{R}_{+}^{d}$ followed by projection onto the $\ell_{1}$-ball $\mathcal{B}_{1}(s) \triangleq\left\{\mathbf{w} \in \mathbb{R}^{d}:\|\mathbf{w}\|_{1} \leq s\right\}$. Both steps can be efficiently implemented. In particular, we can use the method in Duchi et al. [18, Section 4] to perform the second step.

Note that Algorithm 1 ensures that the objective value of (3) does not increase in each iteration $t$. When the relative change in the weight vector $\mathbf{w}$ (measured in $\ell_{1}$-norm) falls below a threshold (e.g., $10^{-4}$ ), we terminate our algorithm.

\subsection{Choice of adjacency matrix and other parameters}

The adjacency matrix $\mathbf{A}$ (which defines the Laplacian $\mathbf{L}$ ), in many cases, can be chosen based on domain knowledge: for image data, $\mathbf{A}$ can be chosen as a binary matrix such that $a_{i j}=1$ if the Euclidean distance between pixels $i$ and $j(i \neq j)$ equals one and $a_{i j}=0$ otherwise. For text data, the adjacency between words can be obtained from lexical databases, e.g., WordNet [29]. For gene expression data, the relation between genes can be obtained from gene pathway databases, e.g., KEGG [30]. Apart from this approach, the adjacency matrix $\mathbf{A}$ can also be learned from data. A simple approach would be to compute $\mathbf{A}$ as the empirical correlation coefficient matrix of all the features (possibly with thresholding). Other 


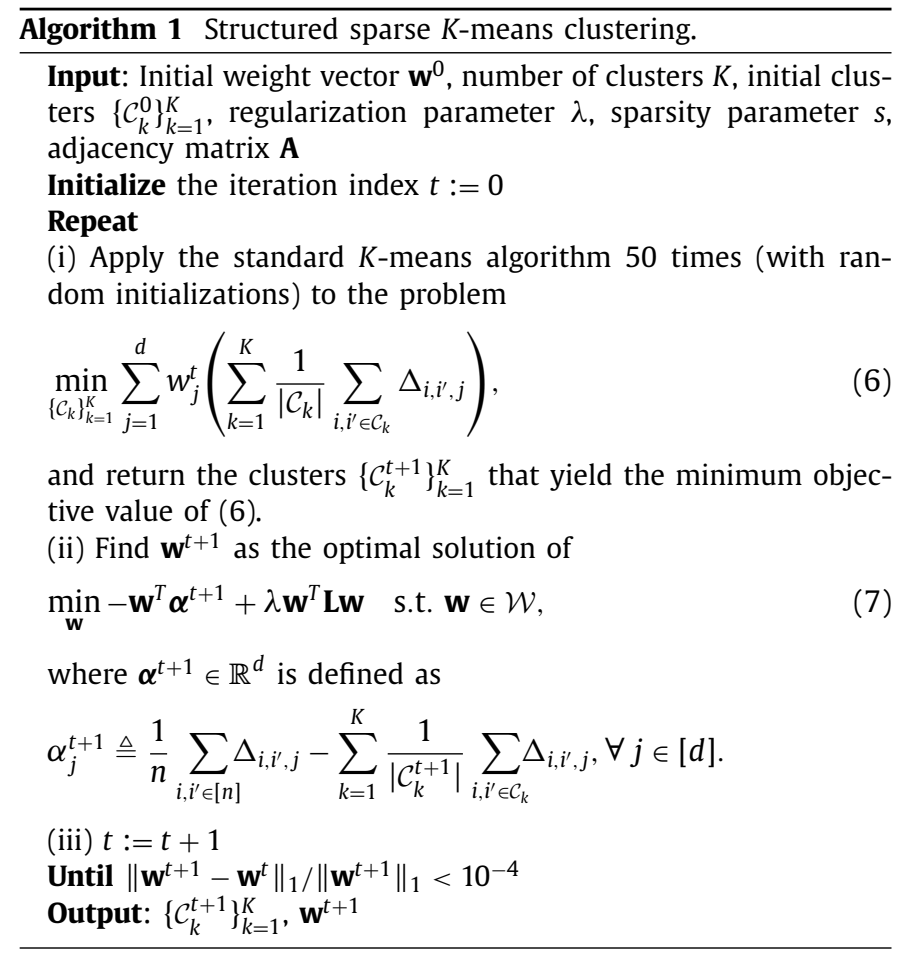

more sophisticated learning approaches include Daitch et al. [14], Dong et al. [17], Leonardi et al. [24], Leonardi and Ville [25].

Next we describe the choice of other parameters. Similar to previous works $[19,28,46]$, we assume that the number of clusters $K$ is known a priori. (Note that there also exists a large volume of literatures $[3,33,38,42,43]$ on the selection of $K$.) The choice of parameters $\lambda$ and $s$ has been a long-standing question, since their values are largely data-dependent. Indeed, so far there lacks an effective yet computationally efficient method in the literature. Two popular parameter selection methods include cross validation [36] and the method of gap statistics (MGS) $[43,46]$. However, both methods are computationally intensive and sometimes yield suboptimal results (in terms of clustering accuracy). Therefore, when comparing different algorithms, we use two methods to choose the values of $\lambda$ and $s$ : grid search (i.e., finding the parameters that yield highest clustering accuracy) and MGS, as suggested in Witten and Tibshirani [46].

\subsection{Extension to non-Euclidean loss}

Our algorithm can be easily extended to the case where the loss measure $\Delta_{i, i^{\prime}, j}$ is beyond the squared Euclidean distance. Indeed, we can define $\Delta_{i, i^{\prime}, j} \triangleq \bar{\delta}\left(x_{i, j}, x_{i^{\prime}, j}\right)$, where $\bar{\delta}: \mathbb{R} \times \mathbb{R} \rightarrow \mathbb{R}_{+}$is a general loss measure. In this case, only step (i) in Algorithm 1 needs to be modified. There are many instances of $\bar{\delta}$ such that (6) can be approximately solved in an efficient way. An important example would be the family of Bregman divergences [12], which includes the squared Euclidean distance as a special case. In this case, the Bregman $K$-means clustering algorithm $[6,26]$ can be readily used in step (i).

\section{Numerical experiments}

We consider clustering tasks on gene expression data and face images respectively. For each task, we compare the performance of our method against the classical $K$-means and the sparse $K$ means [46] methods, in terms of both (learned) feature weights
Table 1

RI and FBC of clustering results by different methods on the Leukemia gene expression dataset. In bold-the best performance amongst all the methods.

\begin{tabular}{llllll}
\hline & K-means & Sp. K-means $(\mathrm{S})$ & Ours $(\mathrm{S})$ & Sp. K-means $(\mathrm{G})$ & Ours $(\mathrm{G})$ \\
\hline RI & 0.53 & 0.87 & $\mathbf{0 . 9 7}$ & 0.87 & $\mathbf{0 . 9 7}$ \\
FBC & 0.55 & 0.79 & $\mathbf{0 . 9 5}$ & 0.79 & $\mathbf{0 . 9 5}$ \\
\hline
\end{tabular}

and clustering accuracy. For all the experiments, we measure clustering accuracy in terms of both Rand Index (RI) and FBCubed measure (FBC). Between them, RI [32] indicates the relative frequency of agreements between the learned pairs of items and the ground truth. FBC [2] is the average of BCubed precision and recall [5] that satisfy a set of formal constraints in Amigó et al. [2].

\subsection{Clustering on gene expression data}

We performed clustering on the popular Leukemia dataset [20], which contains gene expression values of two groups of patients. The first group consists of patients with lymphoblastic leukemia and the second with myeloid leukemia. Following Tibshirani et al. [41], we selected $d=1000$ gene expression values with the largest empirical variance as features. To obtain the (binary) adjacency matrix A, we first computed the empirical correlation coefficient $c_{i j}$ between features $i$ and $j$, for any $i, j \in[d]$. Then we set $a_{i j}=1$ if $c_{i j} \geq 0.5$ and $a_{i j}=0$ otherwise. To select the parameters $\lambda$ and $s$ in (3), we tested $\lambda \in\left\{10^{-4}, 10^{-3.5}, \ldots, 10^{2}\right\}$ and $s \in\left\{2^{0}, 2^{0.5}, \ldots, 2^{6}\right\}$ and reported the values that yielded the highest clustering accuracy. The parameter $\bar{s}$ in the sparse $K$-means method (2) was chosen in the same way as $s$. Besides grid search, we also used MGS to select these parameters for our method and sparse $K$-means.

The clustering accuracy results of $K$-means, sparse $K$-means and our method are shown in Table 1 respectively. In both tables, ' $S$ ' denotes the results given by the parameters (i.e., $\lambda, s$ and $\bar{s}$ ) chosen via grid search and ' $G$ ' denotes those chosen via MGS. From Table 1, in terms of both RI and FBC, we observe that (i) the two parameter selection methods yield the same clustering accuracy for both sparse $K$-means and our method and (ii) the clustering accuracy of our method is significantly higher than the other two.

Next we compare the biological significance of the gene weights returned by sparse $K$-means and our method. To do so, we employed the popular Gene Set Enrichment Analysis (GSEA) method [37]. This method determines whether the identified prominent genes (i.e., genes with large weights) are enriched in a set of predefined terms. Each term is defined as a collection of genes that are related to a biological process. By convention, we used the terms from the Hallmark gene database.

For each term, we used GSEA to compute a measure called false discovery rate (FDR), based on the gene weights returned by sparse $\mathrm{K}$-means or our method. (For a brief description of this procedure, we refer readers to the Appendices Section A.1.) An FDR that falls below 0.05 indicates that the identified genes are enriched in this term.

For all the terms, the results computed from the gene weights returned by sparse $K$-means and our method are shown in Table 2, where the parameters are chosen via grid search. From Table 2, we observe that the prominent genes identified by our method are enriched in 12 terms, while those identified by sparse $K$-means fail to be enriched in any term. According to the descriptions of the 12 terms [22], many are related to the leukemia disease, such as HM_COMPLEMENT and HM_APOPTOSIS. (For completeness, we include such descriptions in Appendices Section A.2.) This shows our method can indeed discover biologically meaningful genes that are relevant to leukemia. As a result, our method renders better clustering accuracy compared to sparse $K$-means. 
Table 2

FDRs computed from the gene weights returned by sparse $K$ means and our method via GSEA on the Leukemia dataset. In bold-the significant terms of our method (FDR $<0.05$ ).

\begin{tabular}{|c|c|c|}
\hline Enrichment term & Ours & Sp. K-means \\
\hline HM_COMPLEMENT & 0.000 & 1.000 \\
\hline HM_TNFA_SIGNALING_VIA_NFKB & 0.000 & 1.000 \\
\hline HM_HYPOXIA & 0.005 & 1.000 \\
\hline HM_APOPTOSIS & 0.012 & 1.000 \\
\hline HM_HEME_METABOLISM & 0.017 & 1.000 \\
\hline HM_EPITHELIAL_MESENCHYMAL & 0.022 & 1.000 \\
\hline HM_KRAS_SIGNALING_UP & 0.030 & 1.000 \\
\hline HM_INFLAMMATORY_RESPONSE & 0.032 & 1.000 \\
\hline HM_UV_RESPONSE_UP & 0.041 & 1.000 \\
\hline HM_INTERFERON_GAMMA & 0.042 & 1.000 \\
\hline HM_ADIPOGENESIS & 0.045 & 1.000 \\
\hline HM_REACTIVE_OXIGEN_SPECIES & 0.046 & 1.000 \\
\hline HM_IL2_STAT5_SIGNALIN̄G & 0.060 & 1.000 \\
\hline HM_P53_PATHWAY & 0.066 & 1.000 \\
\hline HM_ALLOGRAFT_REJECTION & 0.090 & 1.000 \\
\hline HM_XENOBIOTIC_METABOLISM & 0.103 & 1.000 \\
\hline HM_PI3K_AKT_MTOR_SIGNALING & 0.119 & 1.000 \\
\hline HM_FATTY_ACID_METABOLISM & 0.130 & 1.000 \\
\hline HM_MTORC1_SIGNALING & 0.194 & 1.000 \\
\hline HM_GLYCOLYSIS & 0.259 & 1.000 \\
\hline HM_ESTROGEN_RESPONSE_LATE & 0.387 & 1.000 \\
\hline HM_INTERFERON_ALPHA_RESPONSE & 0.602 & 1.000 \\
\hline HM_MYOGENESIS & 0.605 & 1.000 \\
\hline HM_APICAL_JUNCTION & 0.885 & 1.000 \\
\hline HM_OXIDATIVE_PHOSPHORYLATION & 0.972 & 1.000 \\
\hline HM_G2M_CHECKPOINT & 0.979 & 1.000 \\
\hline HM_DNA_REPAIR & 0.997 & 1.000 \\
\hline HM_UNFOLDED_PROTEIN_RESPONSE & 1.000 & 1.000 \\
\hline
\end{tabular}

In addition to the leukemia dataset, in Table 3, we also present the results of clustering accuracy given by $K$-means, sparse $K$-means and our method on six other gene expression datasets used in Jin and Wang [23], with number of classes ranging from two to five. From Table 3, we observe that for both parameter selection methods (i.e., grid search and MGS), our method consistently outperforms the other two on all the six datasets, in terms of both RI and FBC measures.

\subsection{Clustering on face images}

We next compare the clustering performance of three methods on the ORL face dataset [35]. This dataset consists of face images of 40 subjects, with ten images per subject. Each image has a dimension of $19 \times 19$ pixel $^{2}$. To construct the adjacency matrix $\mathbf{A}$, we set $a_{i j}=1$ if pixels $i$ and $j$ share a common edge and $a_{i j}=0$ otherwise. Therefore, pixel neighbours are more likely to share similar weights. The parameters $\lambda, s$ and $\bar{s}$ for sparse $K$-means and our method were selected in the same way as in Section 4.1, i.e., via grid search and MGS. For the interpretability of clustering results, we did not directly cluster the 40 subjects. Instead, we clustered subjects in groups of three and four respectively. Thus in total we had $C(40,3)$ combinations in the three-subject case and $C(40,4)$ combinations in the four-subject case.

We use two approaches to compare the clustering accuracy of our method against $K$-means and sparse $K$-means, for both threeand four-subject cases. We illustrate the comparison between our method and $K$-means on the three-subject case in terms of RI. (Such a comparison procedure can be applied similarly to the four-subject case and the FBC measure.) For each combination $i \in$ $\{1, \ldots, \Gamma\}$, where $\Gamma \triangleq C(40,3)$, we first subtract the RI of $K$-means from ours to obtain their difference and denote it by $\delta_{i}$. Our first approach involves averaging $\left\{\delta_{i}\right\}_{i=1}^{\Gamma}$ to obtain the improved average RI by our method upon $K$-means. To complement this approach, we also conduct the Wilcoxon signed-rank test [45] to the pairs of RIs obtained by our method and $K$-means on all the combinations. As

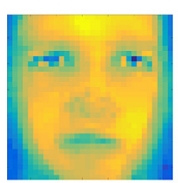

Subj. 1

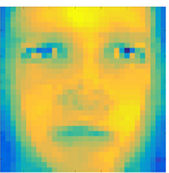

Subj. 1

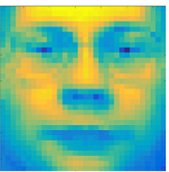

Subj. 4
Subj. 6

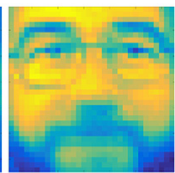

Subj. 28

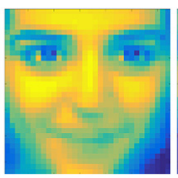

Subj. 10

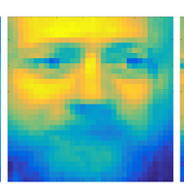

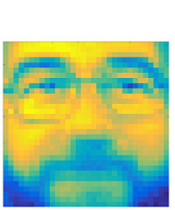

Subj. 28

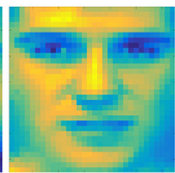

Subj. 30

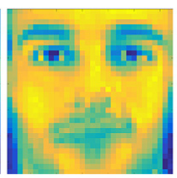

Subj. 16
Sp. $K$-means

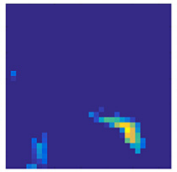

$\mathrm{RI}=0.74$ $\mathrm{FBC}=0.79$

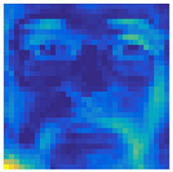

$\mathrm{RI}=0.89$ $\mathrm{FBC}=0.93$

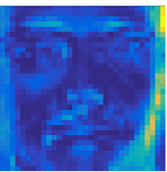

$\mathrm{RI}=0.53$

$\mathrm{FBC}=0.58$
Ours

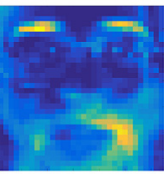

$\mathrm{RI}=1.00$

$\mathrm{FBC}=1.00$

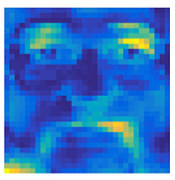

$\mathrm{RI}=1.00$

$\mathrm{FBC}=1.00$

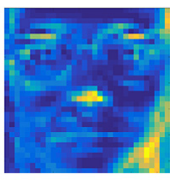

$\mathrm{RI}=0.72$

$\mathrm{FBC}=0.73$
Fig. 2. Comparisons of pixel weights learned by sparse $K$-means and our method in the three-subject case. For each row, from left to right, the first three figures show the average face images of each subject. The last two figures show the pixel weights learned by sparse $K$-means and our method respectively. RI and FBC obtained from either method are also labeled.

shown in Demšar [15], this nonparametric hypothesis test is more robust than the paired $t$-test against the normality assumption in the population. Denote the median of $\left\{\delta_{i}\right\}_{i=1}^{\Gamma}$ as $\delta_{\text {med }}$. We then formulate the null hypothesis $H_{0} \triangleq\left\{\delta_{\text {med }}=0\right\}$ and alternative hypothesis $H_{1} \triangleq\left\{\delta_{\text {med }}>0\right\}$. We reject $H_{0}$ if the resulting $p$-value falls below 0.05 .

The results of improved average RI and $p$-value returned by the Wilcoxon test are shown in Table 4. In terms of the average RI, we observe that our method consistently improves upon the other two methods. In addition, the $p$-values obtained in all the cases are extremely close to zero. This shows that compared to the other two methods, the improvement on the clustering accuracy made by our method is statistically significant.

In addition, the improved average FBC and $p$-value returned by the Wilcoxon test are shown in Table 5. Indeed, we have similar observations to those in Table 4.

We next compare the pixel weights learned by sparse $K$-means and our method in the three-subject case. Three representative combinations are selected and shown in Fig. 2. We observe that for each combination, compared to sparse $K$-means, our method can identify more distinguishable (facial) features. For example, in the first row, the three subjects have distinct eyebrows, which can effectively distinguish them. Indeed, this prominent feature is successfully discovered by our method. In contrast, it is missed by sparse $K$-means. As a result, our method achieves a much higher clustering accuracy (measured in terms of RI and FBC) than sparse $K$-means. Similar observations can be made about the pixel weights learned in the four-subject case (shown in Fig. 3).

\section{Conclusion and future work}

In this work, we have proposed a structured sparse $K$-means clustering algorithm that not only learns the clusters accurately, but also effectively discovers the relevant features (for clustering) that are both sparse and interpretable.

As future work, we aim to develop more efficient methods to choose the regularization weights $\lambda$ and sparsity level $s$ in (3). 
Table 3

Clustering results of different methods on other gene expression datasets, in terms of (a) RI and (b) FBC. In bold-the best performance amongst all the methods.

\begin{tabular}{llllll}
\hline Datasets & K-means & Sp. K-means $(S)$ & Ours $(S)$ & Sp. $K$-means $(G)$ & Ours $(G)$ \\
\hline Brain & 0.83 & 0.87 & $\mathbf{0 . 9 2}$ & 0.85 & $\mathbf{0 . 8 8}$ \\
Colon & 0.73 & 0.77 & $\mathbf{0 . 8 0}$ & 0.77 & $\mathbf{0 . 8 0}$ \\
Leukemia2 & 0.95 & $\mathbf{0 . 9 7}$ & $\mathbf{0 . 9 7}$ & $\mathbf{0 . 9 5}$ & $\mathbf{0 . 9 5}$ \\
Lymphoma & 0.75 & 0.97 & $\mathbf{1 . 0 0}$ & 0.97 & $\mathbf{1 . 0 0}$ \\
Prostate & 0.51 & 0.52 & $\mathbf{0 . 5 3}$ & 0.52 & $\mathbf{0 . 5 3}$ \\
SRBCT & 0.64 & 0.77 & $\mathbf{0 . 8 6}$ & 0.71 & $\mathbf{0 . 7 3}$ \\
(a) RI & & & & & \\
\hline Datasets & K-means & Sp. K-means $(S)$ & Ours $(S)$ & Sp. $K$-means $(G)$ & Ours $(G)$ \\
\hline Brain & 0.62 & 0.74 & $\mathbf{0 . 8 4}$ & 0.67 & $\mathbf{0 . 7 6}$ \\
Colon & 0.75 & 0.78 & $\mathbf{0 . 8 1}$ & 0.78 & $\mathbf{0 . 8 1}$ \\
Leukemia2 & 0.95 & 0.95 & $\mathbf{0 . 9 7}$ & $\mathbf{0 . 9 5}$ & $\mathbf{0 . 9 5}$ \\
Lymphoma & 0.75 & 0.97 & $\mathbf{1 . 0 0}$ & 0.97 & $\mathbf{1 . 0 0}$ \\
Prostate & 0.56 & $\mathbf{0 . 6 0}$ & $\mathbf{0 . 6 0}$ & $\mathbf{0 . 6 0}$ & $\mathbf{0 . 6 4}$ \\
SRBCT & 0.47 & 0.75 & $\mathbf{0 . 7 9}$ & 0.59 & \\
(b) FBC & & & & & \\
\hline
\end{tabular}

Table 4

Improved average RI and $p$-value from Wilcoxon test of our method vs. $K$-means and sparse $K$-means on the ORL dataset.

\begin{tabular}{llll}
\hline & & Ours vs. K-means & Ours vs. Sp. K-means \\
\hline 3-subj. & Improved Ave. RI (S) & 0.07 & 0.02 \\
& $p$-value (S) & 0 & $1 \mathrm{e}-98$ \\
& Improved Ave. RI (G) & 0.06 & 0.04 \\
4-subj. & $p$-value (G) & 0 & 0 \\
& Improved Ave. RI (S) & 0.05 & 0.02 \\
& $p$-value (S) & 0 & 0 \\
& Improved Ave. RI (G) & 0.05 & 0.04 \\
& $p$-value (G) & 0 & 0 \\
\hline
\end{tabular}

Table 5

Improved average FBC and $p$-value from Wilcoxon test of our method vs. $K$-means and sparse $K$-means on the ORL dataset.

\begin{tabular}{llll}
\hline & & Ours vs. K-means & Ours vs. Sp. K-means \\
\hline \multirow{2}{*}{ 3-subj. } & Improved Ave. FBC (S) & 0.10 & 0.004 \\
& $p$-value (S) & 0 & $1 \mathrm{e}-15$ \\
& Improved Ave. FBC (G) & 0.06 & 0.05 \\
& $p$-value (G) & 0 & 0 \\
4-subj. & Improved Ave. FBC (S) & 0.16 & 0.03 \\
& $p$-value (S) & 0 & 0 \\
& Improved Ave. FBC (G) & 0.07 & 0.04 \\
& $p$-value (G) & 0 & 0
\end{tabular}

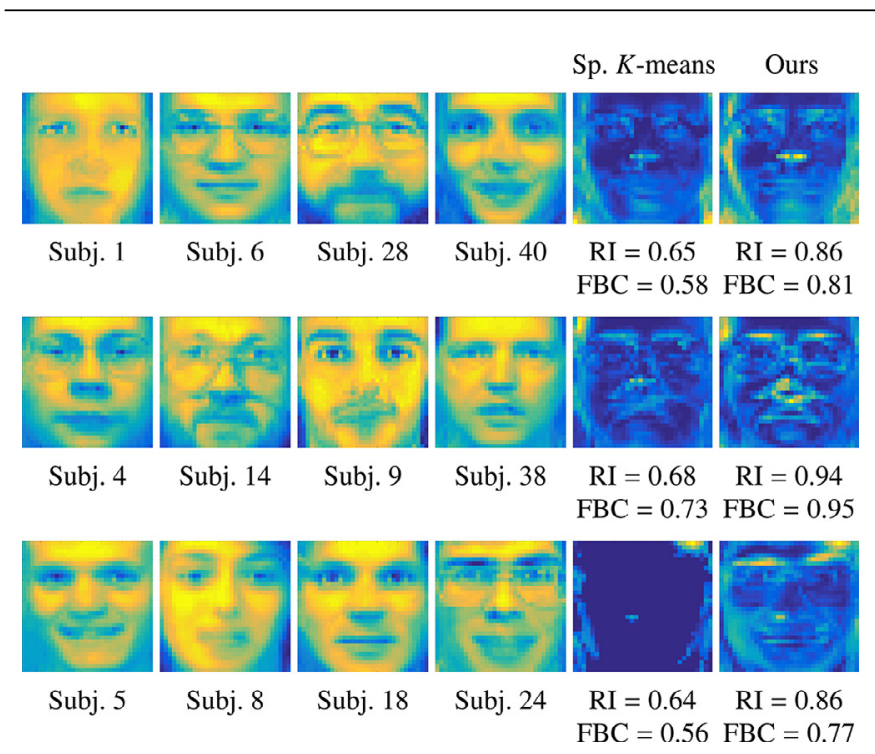

Fig. 3. Comparisons of pixel weights learned by sparse $K$-means and our method in the four-subject case. Descriptions are similar to those for Fig. 2.
Such a parameter selection problem has been largely open in the literature. Besides, we also plan to investigate efficacious ways to learn the adjacency matrix $\mathbf{A}$ from data, under the situations where domain knowledge is not readily available. We believe this will greatly enhance the applicability of our clustering algorithm.

\section{Conflict of Interest}

There is no conflict of interest on our side.

\section{Appendix A}

\section{A.1. Description of GSEA}

This section serves as a brief description of GSEA. For further details, we refer readers to Subramanian et al. [37].

GSEA [37] is a popular knowledge-based tool for interpreting the results of genome-wide expression data analysis. Let $\mathcal{L} \triangleq$ $\left(g_{1}, \ldots, g_{d}\right)$ be a list of genes ranked in descending order according to their weights $\left\{w_{i}\right\}_{i=1}^{d}$ (which are computed by sparse $K$-means or our method). In addition, let $\mathscr{H} \triangleq\left\{\mathcal{S}_{1}, \ldots, \mathcal{S}_{M}\right\}$ be the set of enrichment terms. For each $j \in[M]$, the enrichment term $\mathcal{S}_{j}$ is a collection of genes systematically defined based on prior biological knowledge, e.g., genes located in the same biological pathway. (Note that $\left|\mathcal{S}_{j}\right| \leq d-1$, for any $j \in[M]$.) The purpose of GSEA is to determine whether the genes in $\mathcal{L}$ are significantly correlated with the enrichment terms in $\mathscr{H}$. GSEA consists of three steps, which are described in the sequel.

\section{A.1.1. Calculation of the enrichment score (ES)}

Let $\mathcal{S}$ be any enrichment term in $\mathscr{H}$. For any $i \in[d]$, the ES of the $i$-th gene in $\mathcal{L}$, i.e., $g_{i}$, with respect to (w.r.t.) $\mathcal{S}$ is

$\mathrm{ES}_{i}(\mathcal{S}) \triangleq \sum_{g_{j} \in \mathcal{S}, j \leq i} \frac{\left|w_{j}\right|}{\sum_{g_{j} \in \mathcal{S}}\left|w_{j}\right|}-\frac{\left|\left\{j \in[i]: g_{j} \notin \mathcal{S}\right\}\right|}{d-|\mathcal{S}|}$

Accordingly, the ES of $\mathcal{L}$ w.r.t. $\mathcal{S}$ is defined as

$\mathrm{ES}(\mathcal{S}) \triangleq \max _{i \in[d]} \operatorname{ES}_{i}(\mathcal{S})$

Intuitively, $\operatorname{ES}(\mathcal{S})$ indicates the degree to which $\mathcal{S}$ is represented in the top of the list $\mathcal{L}$. A higher $\operatorname{ES}(\mathcal{S})$ corresponds to a higher degree of representation of $\mathcal{S}$ in $\mathcal{L}$.

\section{A.1.2. Estimation of statistical significance of ES}

We estimate the statistical significance of the enrichment score $\operatorname{ES}(\mathcal{S})$ using a permutation-based hypothesis test. Let $\left\{\mathcal{L}_{\text {perm }}^{q}\right\}_{q=1}^{Q}$ be 
Table A1

Descriptions of the significant enrichment terms in Table 2.

\begin{tabular}{ll}
\hline Enrichment terms & Descriptions \\
\hline HM_COMPLEMENT & Genes encoding components of the complement system (part of the innate immune system) \\
HM_TNFA_SIGNALING_VIA_NFKB & Genes regulated by NF-kB in response to TNF \\
HM_HYPOXIA & Genes up-regulated in response to low oxygen levels (hypoxia) \\
HM_APOPTOSIS & Genes mediating programmed cell death (apoptosis) by activation of caspases \\
HM_HEME_METABOLISM & Genes involved in metabolism of heme and erythroblast differentiation \\
HM_EPITHELIAL_MESENCHYMAL_TRANSITION & Genes defining epithelial-mesenchymal transition, as in wound healing, fibrosis and metastasis \\
HM_KRAS_SIGNALING_UP & Genes up-regulated by KRAS activation \\
HM_INFLAMMATORY_RESPONSE & Genes defining inflammatory response \\
HM_UV_RESPONSE_UP & Genes up-regulated in response to ultraviolet (UV) radiation \\
HM_INTERFERON_GAMMA_RESPONSE & Genes up-regulated in response to IFNG \\
HM_ADIPOGENESIS & Genes up-regulated during adipocyte differentiation (adipogenesis) \\
HM_REACTIVE_OXIGEN_SPECIES_PATHWAY & Genes up-regulated by reactive oxigen species (ROS) \\
\hline
\end{tabular}

a sequence of independently and randomly permuted versions of $\mathcal{L}$. For each $q \in[Q], \mathrm{ES}_{\text {null }}^{q}(\mathcal{S})$ is defined as the ES of the list $\mathcal{L}_{\text {perm }}^{q}$ w.r.t. $\mathcal{S}$. (See Section A.1.1 for details.) The statistical significance of $\operatorname{ES}(\mathcal{S})$ is given by the $p$-value such a hypothesis test, which is calculated as

$p=\frac{1}{Q} \sum_{q=1}^{Q} I_{+}\left(\operatorname{ES}_{\text {null }}^{q}(\mathcal{S})-\mathrm{ES}(\mathcal{S})\right)$,

In (10), $I_{+}$denotes the indicator function of $\mathbb{R}_{+}$, i.e., $I_{+}(a)=1$ if $a \geq 0$ and 0 otherwise. We set $Q=1000$ in our implementation.

\section{A.1.3. Adjustment of the p-values}

As in Section A.1.2, for each $\mathcal{S}_{j} \in \mathscr{H}(j \in[M])$, we need to perform a hypothesis test to find the statistical significance of its enrichment score $\operatorname{ES}\left(\mathcal{S}_{j}\right)$. We denote the $p$-value of this test by $p_{j}$. Due to multiple hypothesis tests, we adjust the $p$-values $\left\{p_{j}\right\}_{j=1}^{M}$ using the Benjamini-Hochberg procedure [10]. Specifically, we order $\left\{p_{j}\right\}_{j=1}^{M}$ in ascending order, i.e., we find a permutation $\pi$ on $[M]$ such that $p_{\pi(1)} \leq \cdots \leq p_{\pi(M)}$. The FDR corresponding to $p_{\pi(j)}$, denoted as $\xi_{j}$, is given by

$\xi_{j} \triangleq \min \left\{\frac{p_{\pi(j)} M}{j}, \frac{p_{\pi(j+1)} M}{j+1}\right\}, \forall j \in[M-1]$

and $\xi_{M} \triangleq p_{\pi(M)}$. Accordingly, define

$\mathcal{A} \triangleq\left\{j \in[M]: \xi_{j}<0.05\right\}$.

If $\mathcal{A}=\varnothing$, then no $p$-value is significant. Otherwise, define $j^{*} \triangleq$ $\max \mathcal{A}$ and we declare that the $p$-values $\left\{p_{\pi(k)}\right\}_{k=1}^{j^{*}}$ are significant.

\section{A.2. Descriptions of the significant enrichment terms}

We briefly describe the significant enrichment terms in Table 2 in Table A1. From the descriptions, we see that all of the significant terms are indeed biologically related to leukemia.

\section{References}

[1] D. Aloise, A. Deshpande, P. Hansen, P. Popat, Np-hardness of euclidean sum-of-squares clustering, Mach. Learn. 75 (2) (2009) 245-248.

[2] E. Amigó, J. Gonzalo, J. Artiles, F. Verdejo, A comparison of extrinsic clustering evaluation metrics based on formal constraints, Inf. Retr. Boston 12 (4) (2009) 461-486.

[3] R.C. de Amorim, C. Hennig, Recovering the number of clusters in data sets with noise features using feature rescaling factors, Inf. Sci. 324 (2015) 126-145.

[4] E. Arias-Castro, X. Pu, A simple approach to sparse clustering, Comput. Stat. Data Anal. 105 (2017) 217-228.

[5] A. Bagga, B. Baldwin, Entity-based cross-document coreferencing using the vector space model, in: Proc. COLING-ACL, Montreal, Quebec, Canada, 1998, pp. 79-85.

[6] A. Banerjee, S. Merugu, I.S. Dhillon, J. Ghosh, Clustering with Bregman divergences, J. Mach. Learn. Res. 6 (2005) 1705-1749.

[7] M. Belkin, P. Niyogi, Using manifold structure for partially labelled classification, in: Proc. NIPS, 2002, pp. 953-960.
[8] M. Belkin, P. Niyogi, Towards a theoretical foundation for Laplacian-based manifold methods, in: Proc. COLT, Bertinoro, Italy, 2005, pp. 486-500.

[9] M. Belkin, P. Niyogi, V. Sindhwani, Manifold regularization: a geometric framework for learning from labeled and unlabeled examples, J. Mach. Learn. Res. 7 (2006) 2399-2434.

[10] Y. Benjamini, Y. Hochberg Controlling the false discovery rate: a practical and powerful approach to multiple testing, J. R. Stat. Soc. Ser. B (Methodol.) (1995) 289-300.

[11] D.P. Bertsekas, Nonlinear Programming, Athena Scitific, 1999.

[12] L.M. Bregman, The relaxation method of finding the common point of convex sets and its application to the solution of problems in convex programming, USSR Comput. Math. Math. Phys. 7 (3) (1967) 200-217.

[13] F.R.K. Chung, Spectral Graph Theory, American Mathematical Society, 1997.

[14] S.I. Daitch, J.A. Kelner, D.A. Spielman, Fitting a graph to vector data, in: Proc. ICML, 2009, pp. 201-208.

[15] J. Demšar, Statistical comparisons of classifiers over multiple data sets, J. Mach. Learn. Res. 7 (2006) 1-30.

[16] E. Dohmatob, A. Mensch, G. Varoquaux, B. Thirion, Learning brain regions via large-scale online structured sparse dictionary learning, in: Proc. NIPS, Barcelona, Spain, 2016, pp. 4610-4618.

[17] X. Dong, D. Thanou, P. Frossard, P. Vandergheynst, Learning laplacian matrix in smooth graph signal representations, IEEE Trans. Signal Process. 64 (23) (2016) 6160-6173.

[18] J. Duchi, S. Shalev-Shwartz, Y. Singer, T. Chandra, Efficient projections onto the 11-ball for learning in high dimensions, in: Proc. ICML, Helsinki, Finland, 2008, pp. 272-279.

[19] J. Friedman, J. Meulman, Clustering objects on subsets of attributes, J. Royal Stat. Soc. Ser. B 66 (2004) 815-849.

[20] T.R. Golub, D.K. Slonim, P. Tamayo, C. Huard, M. Gaasenbeek, J.P. Mesirov, H. Coller, M.L. Loh, J.R. Downing, M.A. Caligiuri, et al., Molecular classification of cancer: class discovery and class prediction by gene expression monitoring, Science 286 (5439) (1999) 531-537.

[21] L. Grosenick, B. Klingenberg, K. Katovich, B. Knutson, J.E. Taylor, Interpretable whole-brain prediction analysis with graphnet, NeuroImage 72 (2013) 304-321.

[22] GSEA \& MSigDB Team, 2017, Molecular signatures database v6.0, URL: http: //software.broadinstitute.org/gsea/msigdb/index.jsp.

[23] J. Jin, W. Wang, Influential features pca for high dimensional clustering, Ann. Stat. 44 (6) (2016) 2323-2359.

[24] N. Leonardi, J. Richiardi, M. Gschwind, S. Simioni, J.-M. Annoni, M. Schluep, P. Vuilleumier, D.V.D. Ville, Principal components of functional connectivity: a new approach to study dynamic brain connectivity during rest, NeuroImage 83 (2013) 937-950.

[25] N. Leonardi, D.V.D. Ville, Wavelet frames on graphs defined by fmri functional connectivity, in: Proc. ISBI, Chicago, IL, USA, 2011, pp. 2136-2139.

[26] C. Liu, M. Belkin, Clustering with bregman divergences: an asymptotic analysis, in: Proc. NIPS, Barcelona, Spain, 2016, pp. 2351-2359.

[27] U. Luxburg, A tutorial on spectral clustering, Stat. Comput. 17 (4) (2007) 395-416.

[28] J. MacQueen, Some methods for classification and analysis of multivariate observations, in: Proc. Berkeley Symp. Math. Stat. Probab., Berkeley, CA, USA 1967, pp. 281-297.

[29] G.A. Miller, Wordnet: a lexical database for english, Commun. ACM 38 (11) (1995).

[30] H. Ogata, S. Goto, K. Sato, W. Fujibuchi, H. Bono, M. Kanehisa, Kegg: kyoto encyclopedia of genes and genomes, Nucleic Acids Res. 27 (1) (1999) 29-34.

[31] W. Pan, X. Shen, Penalized model-based clustering with application to variable selection, J. Mach. Learn. Res. 8 (2007) 1145-1164.

[32] W.M. Rand, Objective criteria for the evaluation of clustering methods, J. Am. Stat. Assoc. 66 (336) (1971) 846-850.

[33] P.J. Rousseeuw, Silhouettes: a graphical aid to the interpretation and validation of cluster analysis, J. Comput. Appl. Math. 20 (1987) 53-65.

[34] V. Sadhanala, Y.-X. Wang, R.J. Tibshirani, Graph sparsifcation approaches for laplacian smoothing, in: Proc. AISTATS, Cadiz, Spain, 2016.

[35] F. Samaria, A. Harter, Parameterisation of a stochastic model for human face identification, in: Proc. WACV, Sarasota, Florida, USA, 1994, pp. 138-142. 
[36] M. Stone, Cross-validatory choice and assessment of statistical predictions, J. Royal Stat. Soc. 36 (2) (1974) 111-147.

[37] A. Subramanian, P. Tamayo, V.K. Mootha, S. Mukherjee, B.L. Ebert, M.A. Gillette, A. Paulovich, S.L. Pomeroy, T.R. Golub, E.S. Lander, et al., Gene set enrichment analysis: a knowledge-based approach for interpreting genome-wide expression profiles, Proc. Natl. Acad. Sci. 102 (43) (2005) 15545-15550.

[38] C.A. Sugar, G.M. James, Finding the number of clusters in a dataset, J. Amer. Stat. Assoc. 98 (463) (2003) 750-763.

[39] W. Sun, J. Wang, Y. Fang, Regularized k-means clustering of high-dimensional data and its asymptotic consistency, Electron. J. Stat. 6 (2012) 148-167.

[40] W. Tansey, J.G. Scott, A fast and flexible algorithm for the graph-fused lasso, 2015, arXiv: 1505.06475.

[41] R. Tibshirani, M. Saunders, S. Rosset, J. Zhu, K. Knight, Sparsity and smoothness via the fused lasso, J. R. Stat. Soc. Ser. B 67 (1) (2005) 91-108.
[42] R. Tibshirani, G. Walther, Cluster validation by prediction strength, J. Comput. Graph. Stat. 14 (3) (2005) 511-528.

[43] R. Tibshirani, G. Walther, T. Hastie, Estimating the number of clusters in a data set via the gap statistic, J. R. Stat. Soc. B 63 (2) (2001) 411-423.

[44] S. Wang, J. Zhu, Variable selection for model-based high-dimensional clustering and its application to microarray data, Biometrics 64 (2008) 440-448.

[45] F. Wilcoxon, Individual comparisons by ranking methods, Biom. Bull. 1 (6) (1945) 80-83.

[46] D.M. Witten, R. Tibshirani, A framework for feature selection in clustering, J. Amer. Stat. Assoc. 105 (490) (2010) 713-726.

[47] B. Xin, Y. Kawahara, Y. Wang, L. Hu, W. Gao, Efficient generalized fused lasso and its applications, ACM Trans. Intell. Syst. Technol. 7 (4) (2016) 1-22.

[48] D. Zhou, J. Huang, B. Schölkopf, Learning from labeled and unlabeled data on a directed graph, in: Proc. ICML, Bonn, Germany, 2005, pp. 1036-1043. 